\title{
Abnormal hematopoietic phenotypes in Pim kinase triple knockout mice
}

\author{
Ningfei An, Andrew S Kraft and Yubin Kang*
}

\begin{abstract}
Background: Pim (proviral insertion in murine lymphoma) kinases are a small family of constitutively active, highly conservative serine/threonine oncogenic kinases and have 3 members: Pim1, Pim2, and Pim3. Pim kinases are also implicated in the regulation of B- and T- cell responses to cytokines and hematopoietic growth factors. The roles of Pim kinases in the regulation of primitive hematopoietic stem cells (HSCs) are largely unknown.

Methods: In the current study, Pim $1^{-/-} 2^{-/-} 3^{-/-}$triple knockout (TKO) mice were used to determine the role of Pim kinases in hematopoiesis. Peripheral blood hematological parameters were measured in Pim TKO mice and age-matched wild-type (WT) controls. Primary, secondary, and competitive transplantations were performed to assay the long-term repopulating HSCs in Pim TKO mice. In vivo BrdU incorporation assay and ex vivo Ki67 staining and caspase 3 labeling were performed to evaluate the proliferation and apoptosis of HSCs in Pim TKO mice.

Results: Compared to age-matched WT controls, Pim TKO mice had lower peripheral blood platelet count and exhibited erythrocyte hypochromic microcytosis. The bone marrow cells from Pim TKO mice demonstrated decreased hematopoietic progenitor colony-forming ability. Importantly, Pim TKO bone marrow cells had significantly impaired capacity in rescuing lethally irradiated mice and reconstituting hematopoiesis in primary, secondary and competitive transplant models. In vivo BrdU incorporation in long-term HSCs was reduced in Pim TKO mice. Finally, cultured HSCs from Pim TKO mice showed reduced proliferation evaluated by Ki67 staining and higher rate of apoptosis via caspase 3 activation.
\end{abstract}

Conclusions: Pim kinases are not only essential in the hematopoietic lineage cell development, but also important in HSC expansion, self-renewal, and long-term repopulation.

Keywords: Serine/threonine kinase, Pim kinase, Hematopoietic stem cells, Hematopoietic stem cell transplantation, Proliferation, Apoptosis, Knockout mouse

\section{Background}

Pim1, Pim2 and Pim3 belong to a small family of serine/threonine protein kinases and are evolutionarily conserved in multicellular organisms. Pim1 and Pim2 were originally identified from cloning the retroviral integration sites in murine Moloney Leukemia virus (MuLV)-induced lymphomas [1,2]. Pim3 was identified through high throughput retroviral tagging in tumors of c-Myc transgenic mice deficient for Pim1 and Pim2 [3]. Although Pim kinase genes are located on different chromosomes, they encode proteins with a high degree of sequence homology $[4,5]$. Additionally, the functions and expression patterns of

\footnotetext{
* Correspondence: kangy@musc.edu.

Division of Hematology-Oncology, Department of Medicine, Medical University of South Carolina, 86 Jonathan Lucas Street, Hollings Cancer Center Rm\# HO307, Charleston, SC 29425, USA
}

Pim kinases overlap significantly with each other $[4,6,7]$. For example, Pim3 can compensate for the loss of Pim1 and Pim2 in MuLV-induced lymphomagenesis [3]. E $\mu$ MycE $\mu$ Pim2 double transgenic mice develop B cell lymphoid tumors similar to those seen in $\mathrm{E} \mu \mathrm{Myc}-\mathrm{E} \mu \mathrm{Pim} 1$ double transgenic mice $[8,9]$. Pim kinases are constitutively active and play an important role in tumor cell cycle regulation and in cancer cell survival [5].

Over the last two decades, several genetically modified mice were generated to facilitate the studies of the functional roles of Pim kinases. These animal models included E $\mu$-Pim1 transgenic mice [8], $\mathrm{Pim}^{-1-}$ single knockout $(\mathrm{KO})$ mice [10], Pim $2^{-/-}$single $\mathrm{KO}$ mice [11], and $\mathrm{Pim}^{-/-} 2^{-/-} 3^{-/-}$triple KO (TKO) mice [4]. $\mathrm{Pim}^{-/-}$and $\mathrm{Pim} 2^{-/-}$single $\mathrm{KO}$ mice do not show any 
anatomic or developmental defects likely in part due to the functional redundancy and overlap of Pim kinases. Pim single or triple $\mathrm{KO}$ mice are all viable and show subtle hematological changes such as anemia, erythrocyte microcytosis, reduced peripheral $\mathrm{T}$ - and $\mathrm{B}$ - cell numbers, and impaired T- and B- cell responses to IL-2, IL-3 and IL-7 stimulation [4,10]. Grundler, et al. [12] recently found that Pim1 was important in regulating the surface expression of CXCR4 chemokine receptor in hematopoietic stem cells (HSCs). Pim1 phosphorylates serine 339 of the intracellular domain of CXCR4, a site critical for CXCR4 recycling [12]. However, very little is known about the effects of Pim kinases on hematopoiesis and the roles of Pim kinases in the expansion and proliferation of primitive HSCs.

We recently reported a quantitative real-time PCRbased technique for determination of donor cell engraftment in a competitive murine transplantation model [13]. Our PCR method measures the Y chromosome specific gene, i.e., Zfy-1, and can be used for any strain of mouse transplantation models. In the current study, we performed serial transplant experiments and competitive transplant experiments to analyze the hematopoietic phenotypes of Pim TKO mice. We showed that Pim TKO HSCs are deficient in self-renewal and long-term repopulation. These defects are at least in part due to reduced cell proliferation and increased cell apoptosis in the most primitive HSC compartment in Pim TKO mice.

\section{Results}

\section{Thrombocytopenia and erythrocyte hypochromic microcytosis in Pim TKO mice}

To determine the effects of Pim kinases on hematopoiesis, we first measured peripheral blood white blood cells, red blood cells, platelets and hemoglobin in 3 different age groups of Pim TKO mice (i.e., 1-2 months old, 2-4 months old and 6-7 months old). Sex- and age- matched wildtype (WT) control mice were used for comparison. While the total white blood cell counts were comparable between Pim TKO mice and WT controls, the platelet count was significantly reduced in Pim TKO mice (Figure 1A, p <0.01). Consistent with previous reports with Pim deficient mice $[4,10,11]$, Pim TKO mice exhibited erythrocyte hypochromic microcytosis that was characterized by unchanged total hemoglobin level with increased red blood cell count (Figure $1 \mathrm{~B}, 1^{\text {st }}$ and $2^{\text {nd }}$ panel). The erythrocyte hypochromic microcytosis was further confirmed by measuring the mean corpuscular volume $(\mathrm{MCV})$ and the mean corpuscular hemoglobin $(\mathrm{MCH})$ of red blood cells, both of which were significantly reduced in Pim TKO mice (Figure $1 \mathrm{~B}, 3^{\text {rd }}$ and $4^{\text {th }}$ panel, $\left.\mathrm{p}<0.01\right)$. We then performed a detailed peripheral cell subset analysis that quantified the absolute number of peripheral $\mathrm{Gr}-1^{+}$granulocytes, $\mathrm{CD}^{+} \mathrm{T}$ cells, $\mathrm{CD} 4^{+}$cells, $\mathrm{CD}^{+}$cells and $\mathrm{B} 220^{+} \mathrm{B}$ cells [14]. Compared to WT controls, Pim TKO mice had significantly lower number of
$\mathrm{CD}^{+} \mathrm{T}$ cells, predominantly in the $\mathrm{CD}^{+} \mathrm{T}$ helper cell population (Figure $1 \mathrm{C}, \mathrm{p}<0.01$ ). $\mathrm{Gr}-1^{+}$granulocytes were also reduced in Pim TKO mice. These results suggested that Pim TKO mice had impairment in multiple lineages of hematopoietic cells.

\section{Reduced hematopoietic stem/progenitor cell number and colony-forming units in Pim TKO mice}

Pim TKO mice were significantly smaller in size than heterozygous littermates [4]. Interestingly, when we measured the spleen weight and adjusted it to the total body weight, the Pim TKO mice still exhibited significant reduction in the spleen-to-body weight ratio. The spleen-to-body weight ratio was reduced by half in Pim TKO mice compared to sex- and age- matched WT controls (Figure 2A, p <0.01). Additionally, Pim TKO mice had significantly lower number of total bone marrow cells (measured from 2 femurs and 2 tibias of each mouse) (Figure 2B, p < 0.05). Total number of splenocytes was also reduced in Pim TKO mice in comparison to WT controls (Figure $2 \mathrm{C}, \mathrm{p}<0.01$ ). These data suggested a broadly diminished hematopoietic compartment in Pim TKO mice.

We next performed in vitro colony-forming unit (CFU) assay to determine the frequency of hematopoietic stem/ progenitor cells (HSPCs) in the bone marrow (BM) of Pim TKO mice. As shown in Figure 2D, CFUs-granulocyte/ macrophage (CFUs-GM), Burst-forming units-erythrocyte (BFUs-E), and CFUs-granulocyte, erythrocyte, monocyte, and megakaryocyte (CFUs-GEMM) were significantly reduced in Pim TKO mice, demonstrating reduced clonogenic activity of HSPCs in Pim TKO mice.

We also measured the percentage and absolute number of $\mathrm{Lin}^{-} \mathrm{Sca}-1^{+} \mathrm{C}-\mathrm{Kit}^{+}$(LSK) cells in BM (Figure 2E). LSK cells virtually account for all HSPCs in mice. We found that the percentage of LSK cells in the BM of Pim TKO mice was comparable to that in WT controls (Figure 2E). However, since the total BM cell mass was reduced in Pim TKO mice, the absolute number of LSK cells was significantly lower in Pim TKO mice than in WT mice (Figure 2F). To further characterize HSPC population in Pim TKO mice, we measured long-term (LT)-HSCs (CD34 CD135 LSK cells), short-term (ST)-HSCs (CD34CD $135^{+}$LSK cells), and multi-potential progenitor cells (MPPs; CD34 ${ }^{+} \mathrm{CD} 135^{+}$LSK cells) [15]. We found that the percentages of LT-HSCs, ST-HSCs and MPPs were quite similar between Pim TKO mice and WT controls (Figure 2G), although one would expect that their absolute numbers would be decreased in Pim TKO mice.

\section{Reduced self-renewal and long-term repopulating capacity of HSCs in Pim TKO mice}

HSCs have the ability to self-renew and differentiate into all lineages of hematopoietic cells. These functions are best demonstrated using transplantation models in which 


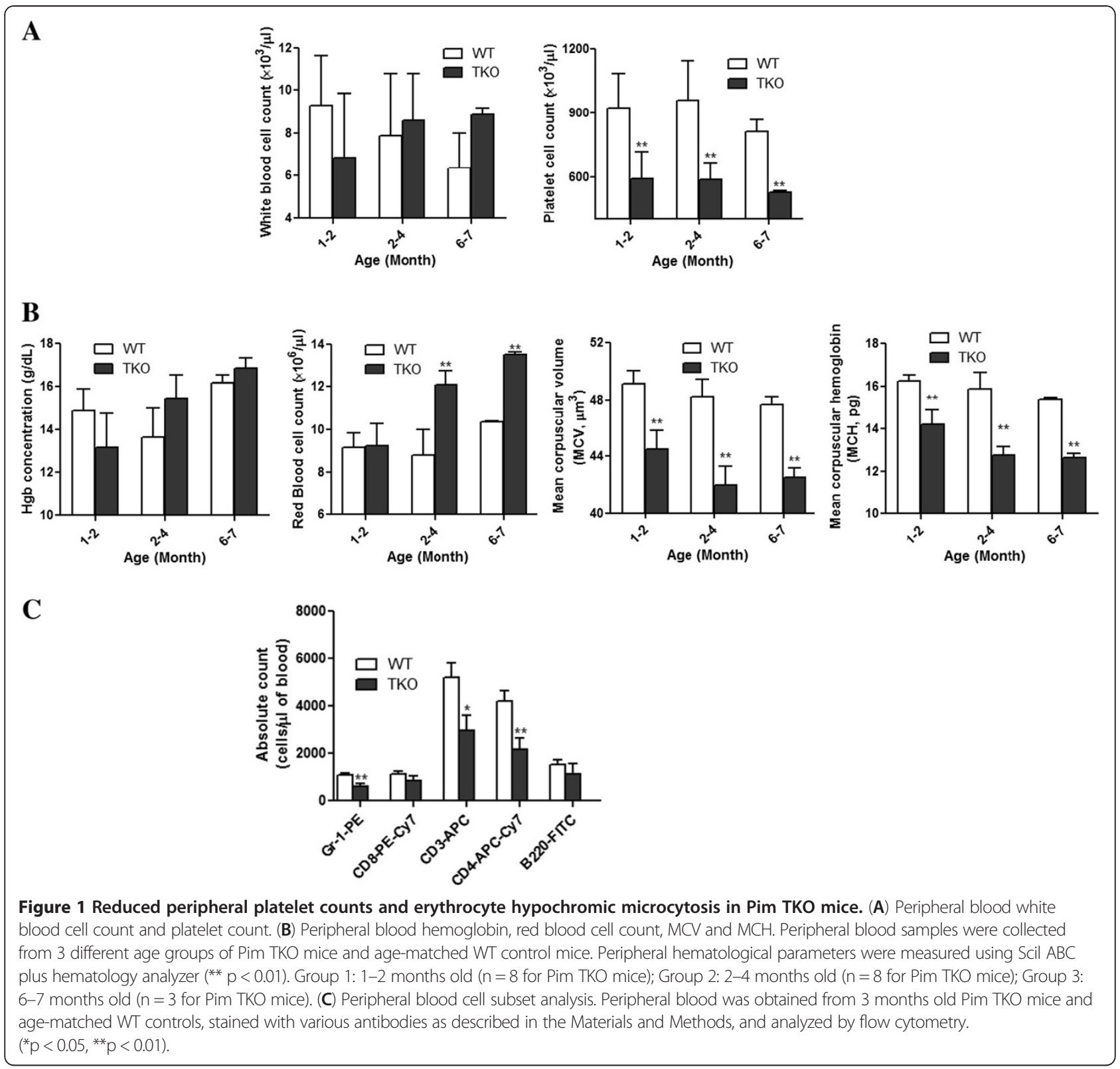

the transplanted HSCs rescue lethally irradiated recipients and reconstitute the whole hematological system. We transplanted lethally irradiated (11 Gy) FVB/J mice with BM cells harvested from Pim TKO mice or sex- and agematched WT controls $\left(5 \times 10^{5}\right.$ cells/recipient mouse). As showed in Figure 3A, the mice transplanted with WT BM cells all survived after transplantation. In contrast, $75 \%$ of the mice transplanted with BM cells from Pim TKO mice died after transplantation. Additionally, those Pim TKO BM transplant recipient mice that survived showed significantly lower peripheral white blood cell counts at various time-points post transplantation (Figure 3B). The platelet count at 3 months post transplantation was also significantly lower in Pim TKO BM transplant recipient mice than that in the mice transplanted with WT BM cells (Figure 3C). The impaired hematological reconstitution seen in Pim TKO primary transplant recipient mice was not due to fewer number of LSK cells transplanted. All mice received equal number of BM cells and the percentages of LSK cells were similar between Pim TKO BM grafts and WT BM grafts (Figure 2E).

To further define the role of Pim kinases on HSC selfrenewal and long-term repopulation, we performed secondary $\mathrm{BM}$ transplantation (Figure 4A-4B). BM cells were harvested from primary $\mathrm{BM}$ transplanted recipient mice at 4 months post transplantation and injected into lethally irradiated female $\mathrm{FVB} / \mathrm{J}$ mice $\left(1 \times 10^{7}\right.$ cells/mouse). At 3 months post transplantation, the secondary transplant mice 


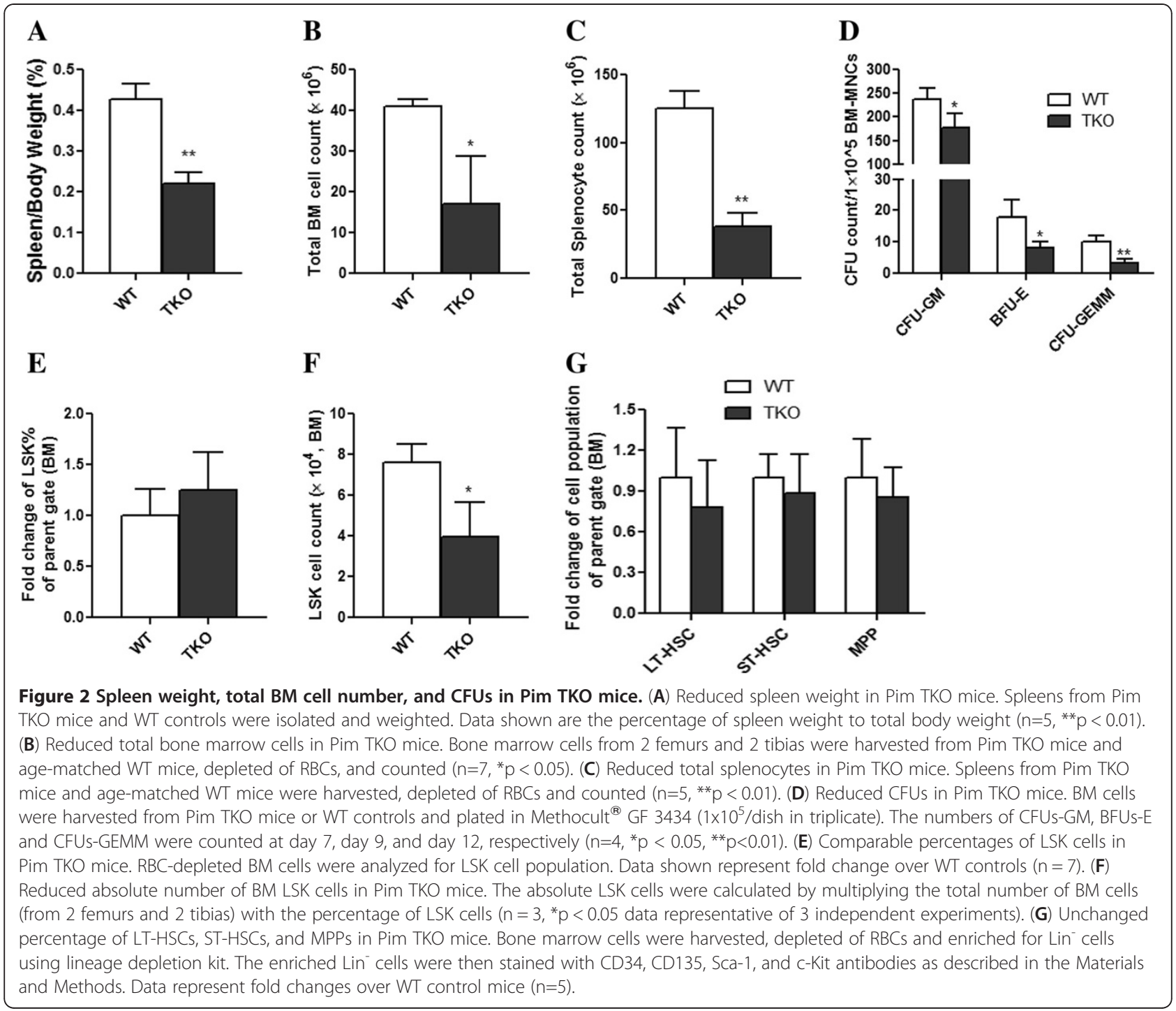

receiving Pim TKO cells had significantly reduced peripheral white blood cell counts (Figure 4A). Donor cell engraftment in $\mathrm{BM}$ was quantified at 4 months post transplantation using a quantitative PCR-based method measuring the sex-determining region $Y(Z f y 1)[13,16]$. We found that the male donor cells engraftment in secondary Pim TKO transplant recipients was significantly lower than that in controls (Figure 4B). Our data demonstrated that the self-renewal potential and the long-term repopulating capacity of HSCs were impaired in Pim TKO mice.

Competitive repopulating capacity of Pim TKO BM cells was also examined. In this series of experiments, $5 \times 10^{5} \mathrm{BM}$ cells from male Pim TKO mice or male WT controls were mixed with $2 \times 10^{5}$ of competitive WT female FVB/J BM cells and transplanted into lethally irradiated female FVB/J recipients. Male donor cell engraftment in peripheral blood at 3 months post transplantation was estimated by quantitative PCR. As shown in Figure 5A, mice transplanted with
Pim TKO BM cells had a lower percentage of donorderived male cells compared to control mice $(\mathrm{p}<0.01)$. These results indicated that Pim TKO BM cells were less efficient and less competent in reconstituting the hematopoietic system than WT BM cells. We also determined donor cell engraftment in three main peripheral blood cell subsets (i.e., Gr- $1^{+}$granulocytes, $\mathrm{T}$ cells and $\mathrm{B}$ cells) in the competitive transplant recipient mice. As shown in Figure 5B, the donor cell engraftment was reduced in all these three cell subsets in competitive transplant recipient mice receiving Pim TKO BM cells, suggesting impairment in multipotential HSCs in Pim TKO mice.

\section{Reduced HSC proliferation and increased apoptosis in Pim TKO mice}

To understand the mechanisms underlying the reduced capacity of Pim TKO HSCs in reconstituting hematopoiesis, we 


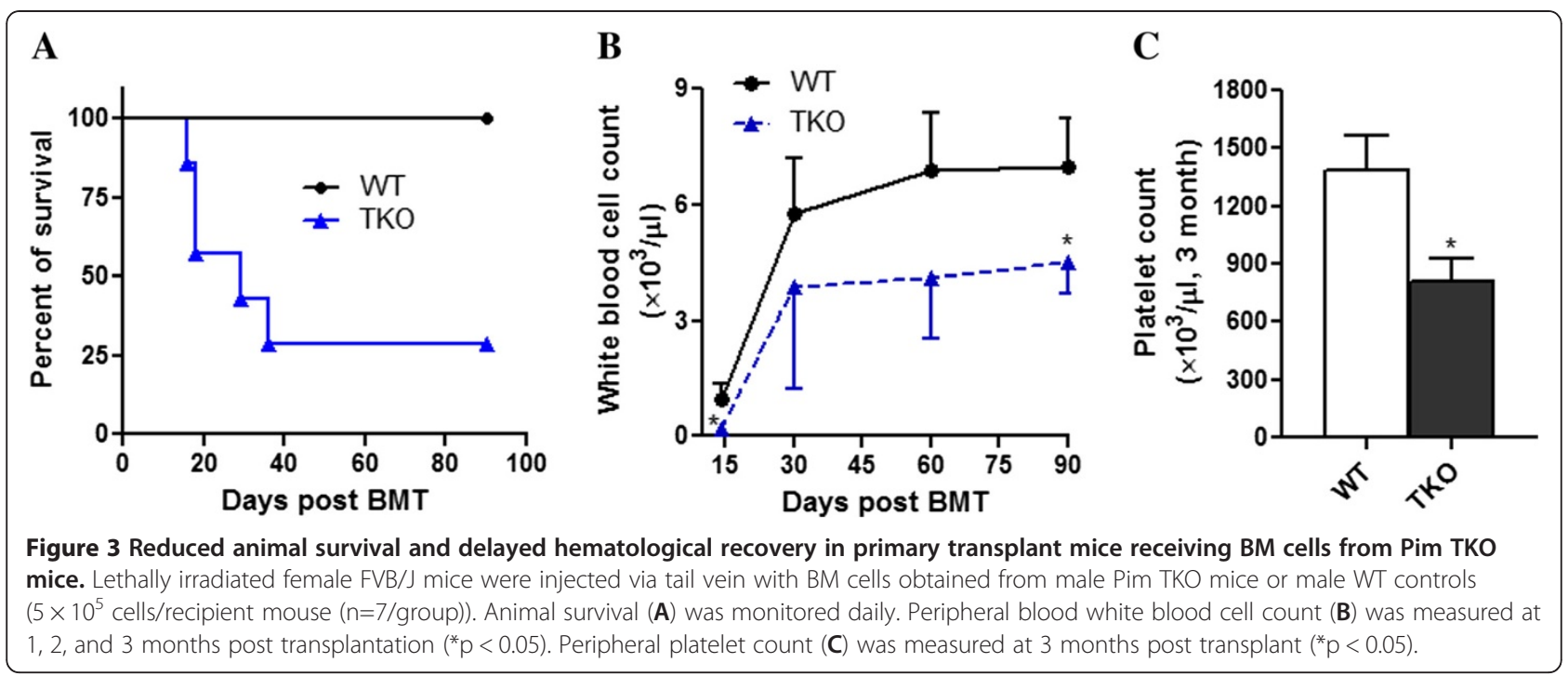

investigated the proliferation status of HSCs in Pim TKO mice. We first measured in vivo BrdU incorporation in LTHSCs, ST-HSCs and MPPs of the mice. We observed significantly lower number of BrdU positive cells in the Pim TKO LT-HSC population (Figure 6A). The BrdU incorporation in ST-HSC and MPP compartments was not affected in Pim TKO mice (Figure 6A). We next measured Ki67 labeling in cultured LSK cells in vitro. We sorted BM LSK cells from Pim TKO mice or WT mice and cultured the cells in StemSpan medium supplemented with growth factors for 48 hours. Consistent with our in vivo BrdU results, we found that the mean fluorescent intensity (MFI) of Ki-67 expression in Pim TKO LSK cells was reduced by $20 \%$ compared to that in WT LSK cells (Figure 6B). These data suggested that Pim TKO HSCs were less proliferative.

To determine the role of Pim kinases in regulating HSC cell survival, we measured cell death and Caspase 3- activation in LSK cells ex vivo. Pim TKO LSK cells and WT LSK cells were cultured in vitro with growth factors for 48 hours. Cell death was measured by live/dead fixable dye and caspase 3- activation was determined by intracellular caspase 3 antibody staining. Compared to WT LSK cells,
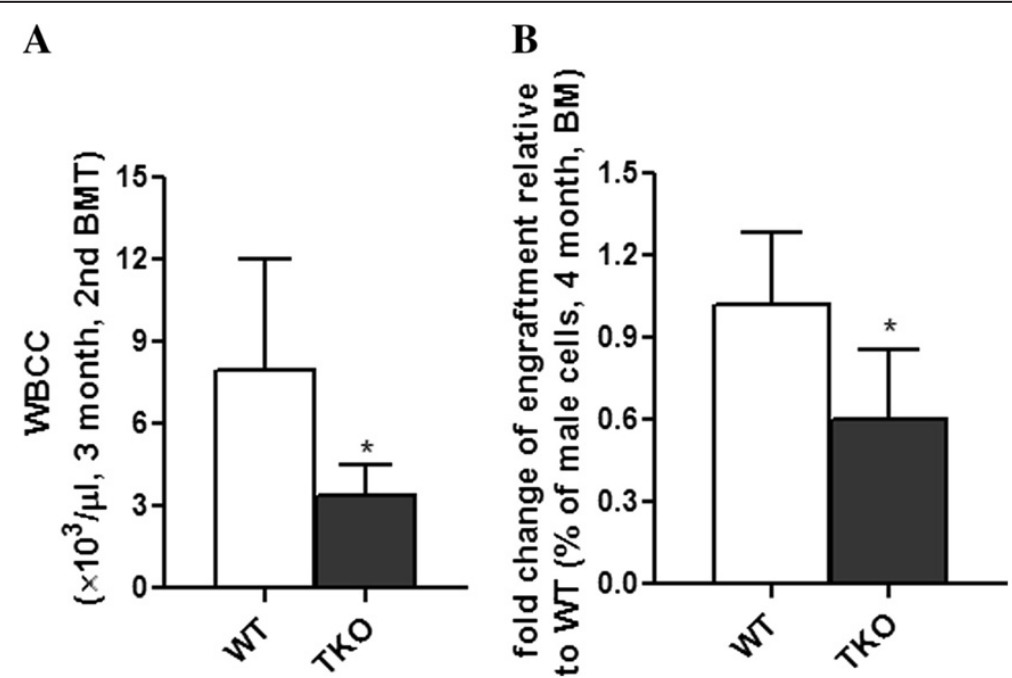

Figure 4 Delayed hematological recovery and reduced donor cell contribution in secondary transplant mice receiving BM cells from Pim TKO primary transplant recipients. BM cells were harvested from primary transplant recipients at 4 months post transplantation and injected into lethally irradiated female FVB recipients $\left(1 \times 10^{7} /\right.$ mouse). (A) Lower peripheral white blood cell counts in Pim TKO secondary transplant recipient mice. Peripheral white blood cell count in the secondary transplant recipient mice was measured at 3 months post transplant ( $n=6$ for WT; $n=3$ for Pim TKO mice, ${ }^{*} p<0.05$ ). (B) Reduced donor cell engraftment in the BM of Pim TKO secondary transplant recipient mice. The secondary transplant recipient mice were sacrificed at 4 months post transplant and BM cells were harvested and analyzed for male donor cell engraftment $\left({ }^{*} \mathrm{p}<0.05\right)$. 

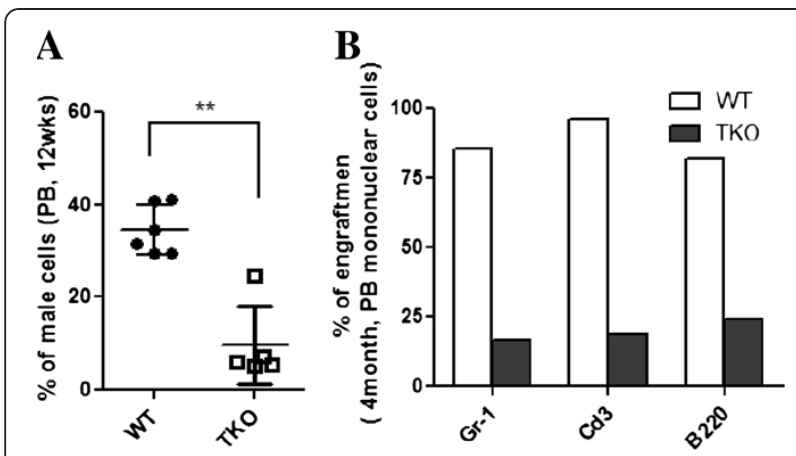

Figure 5 Reduced repopulating capacity of Pim TKO BM cells in competitive transplants. $5 \times 10^{5}$ total BM cells from male Pim TKO mice or WT controls, along with $2 \times 10^{5}$ female competitor BM cells, were transplanted into irradiated female recipients. (A) Reduced engraftment capacity of Pim TKO BM cells. Male donor engraftment in peripheral blood at 12 weeks post transplant was estimated $\left({ }^{* *} p<0.01\right)$. (B) Reduced engraftment in all three main cell subsets with Pim TKO BM cells. Gr-1 $1^{+}$granulocytes, CD3e $\mathrm{e}^{+} \mathrm{T}$ cells and $\mathrm{B} 220^{+}$ $B$ cells were sorted from pooled peripheral blood samples. Genomic DNAs from each population were isolated and the male donor cell contribution in these cell subsets was determined.

Pim TKO LSK cells showed increased rates of cell death (Figure 6C) and caspase 3- activation (Figure 6D).

\section{Discussion}

Hematopoiesis is regulated by many different molecular pathways [17]. In the current study, we examined the role of Pim kinases in regulating the primitive HSCs in mice. We used serial transplant experiments, competitive transplant assay, and in vivo and in vitro proliferation assays to investigate the long-term repopulating HSCs in Pim TKO mice. Our study provides direct evidence for an important role of Pim kinases in hematopoiesis. Our findings are consistent with and support previous observations reported by others [4,10-12]. For instance, we found that Pim TKO mice had reduced body size, displayed erythrocyte microcytosis, and had reduced $\mathrm{T}$ cell numbers. We did not observe significant changes in the peripheral B- cell number. This is also consistent with previous observation by Mikkers, et al., who found that in young Pim TKO mice, peripheral B- cell numbers were reduced whereas in older animals, the B- cell number was unaltered [4]. Importantly, our current study extended our observations beyond previous results. We demonstrated that: 1). Deletion of Pim kinases affects multiple lineages of hematopoietic cells including platelet counts (Figures 1). 2). Deletion of Pim kinases affects the self-renewal and long-term repopulating capacity of HSCs (Figures 3, 4, 5). 3). Deletion of Pim kinases affects the proliferation of the most primitive HSCs in vivo and in vitro (Figure 6). and 4). Deletion of Pim kinases increases apoptotic cell death of HSCs (Figure 6). Our study provides new insights into the roles of Pim kinases in the regulation of HSCs.
The reasons the effects of Pim kinases in HSCs were not previously reported are: 1) Previous studies had been focused on the effects of Pim kinases on T- and B-cells. This seemed logical because overexpression of Pim1 kinase induced clonal T cell lymphoma/leukemia [8]. 2) Pimdeficient mice were generated on $\mathrm{FVB} / \mathrm{J}$ background $[4,10,11]$. FVB/J mice lack readily available surface markers to separate donor-derived cells from congenic recipient-origin cells. This presents a technical challenge in determining the long-term repopulating capacity and self-renewal of HSCs in transplantation models. 3) The percentage of LSK cells in the BM of Pim TKO mice was comparable to that in WT controls, as we showed in Figure 2E. In the absence of serial transplant experiments, this finding may lead investigators to assume that HSC population is unaltered in Pim-deficient mice. The PCR-based method that we reported recently [13] allows us to reliably determine donor cell engraftment in our transplant experiments.

Several published studies suggested a potentially important role of Pim kinases in hematopoiesis and in HSCs. For example, Pim1 is highly expressed in human fetal hematopoietic tissues [18]. Additionally, Pim1 kinase is a key target for HOXA9, a homeoprotein important in hematopoiesis [19]. Pim1 and Pim3 were found to be important in maintaining the self-renewal of mouse embryonic stem cells, and loss of Pim1 and Pim3 led to cell differentiation [20]. Furthermore, overexpression of Pim kinase protected hematopoietic cells from apoptosis [21], and enhanced growth factor- independent survival in myeloid cells $[22,23]$. Recent study by Grundler, et al. [12] suggested that Pim1 kinase was critical in CXCR4 expression and HSC homing. Using transplant models, our study provides direct evidence for an important role of Pim kinases in hematopoiesis.

Pim kinases regulate diverse signal pathways in both hematological and non-hematological malignant cells. Pim kinases promote cell proliferation by regulating enzymes that are important in cell cycle progression, including Cdc25A [24,25] and p27kip1 [26]. Pim kinases regulate cell survival by phosphorylating the apoptotic protein BAD [27] and ASK1 [27]. Furthermore, Pim1 kinase regulates PRAS40 phosphorylation and increases the activities of mammalian target of rapamycin protein kinase [29]. Pim kinase was found to be important in controlling energy metabolism and cell growth [30]. Consistent with these observations in cancer cells, our studies suggest that Pim kinases are important in the regulation of cell proliferation and survival in HSCs.

Our study has important implications. Pim kinases are being investigated as a potential target in the drug development for the treatment of cancer [31]. Several compounds including pan- Pim inhibitors are currently under development and have shown interesting preclinical activities in 

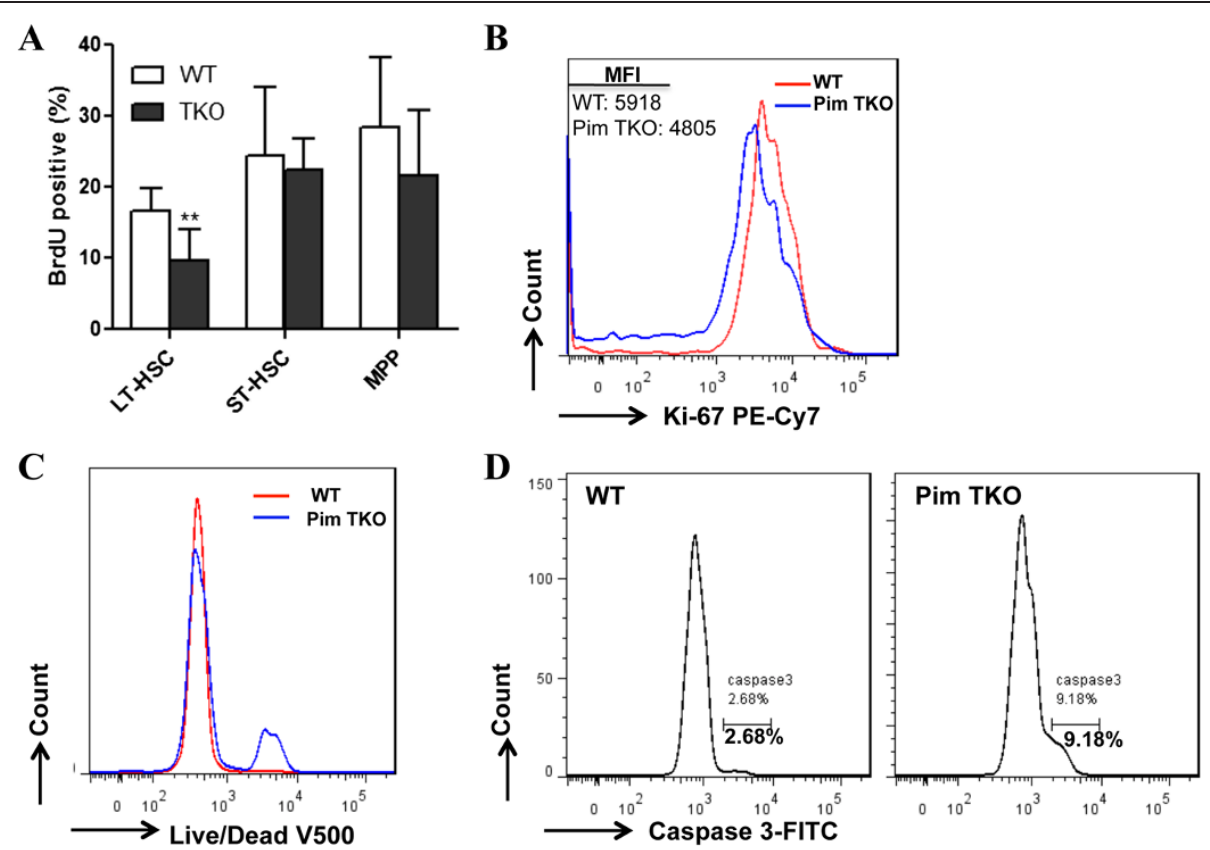

Figure 6 Decreased proliferation and increased apoptosis of Pim TKO HSCs. (A) Decreased in vivo BrdU incorporation in LT-HSCS of Pim TKO mice. Pim TKO mice or WT mice were injected with 2 doses of BrdU, and BM cells were harvested and labeled with antibodies. BrdU-positive cells in LT-HSCs, ST-HSCs, and MPPs were analyzed $\left(n=6,{ }^{* *} P<0.01\right)$. (B) Decreased in vitro Ki67 labeling in Pim TKO LSK cells. LSK cells were sorted from Pim TKO mice or WT mice and cultured in StemSpan medium supplemented with TPO, SCF and Flt3 for 2 days. The cells were then stained with Ki67 antibody. Representative histogram of three independent experiments was shown (MFI: mean florescent intensity). (C) Increased cell death in Pim TKO LSK cells. The cultured LSK cells were stained with Live/Dead dye and representative histogram of three independent experiments was shown. (D) Increased caspase 3-activation in Pim TKO LSK cells. Cultured LSK cells were fixed and stained with caspase 3 antibody. Representative histogram of three independent experiments was shown.

multiple cancer histologies. Clinical data and safety profiles of these inhibitors in human are very limited. Our current studies suggest that it would be important to understand and monitor the potential hematological side effects when using Pim kinase inhibitors.

\section{Conclusions}

We demonstrated that Pim kinases play a fundamental role in HSC regulation. Our findings support the notion that oncogenes are not only important in tumorigenesis, but also involved in normal cell development. Identifying these roles is an important step in developing safe and effective therapeutic agents for the treatment of cancers.

\section{Materials and methods}

Antibodies and reagents

APC-conjugated anti- mouse CD117 antibody (c-Kit, 2B8), APC-H7-conjuagted anti- mouse c-Kit antibody (2B8), PEconjugated anti- mouse Sca-1 antibody (E13-161.7), APCconjugated anti- mouse CD3e (145-2C11), PE-conjugated anti mouse Gr-1 (RB6-8C5), PE-Cy7-conjugated Ki67 antibody (B56), FITC- labeled Caspase3 (C92-605) antibody and FITC-BrdU Flow kit were purchased from BD Pharmingen (San Diego, CA). FITC- conjugated anti- mouse B220 (RA3-6B2); PerCP-eFluor 710- labeled anti- mouse CD135 (A2F10) and eFluor 450- conjugated anti- mouse CD34 (RAM34) antibodies were purchased from eBiosciences (San Diego, CA). Magnetic murine lineage cell depletion kit was purchased from Miltenyi Biotec (Auburn, CA). Aqua Live/dead fixable dye was purchased from Invitrogen (Grand Island, NY).

\section{Mice}

\section{Pim TKO mice}

$\operatorname{Pim} 1^{-1-} 2^{-1-}$ and $\mathrm{Pim} 2^{-/-} 3^{-/-}$double $\mathrm{KO}$ mice were generated by Mikkers, et al. [4] and were a kind gift of Drs Paul B. Rothman (Johns Hopkins University) and Anton Berns (The Netherlands Cancer Institute). Pim $1^{-/-} 2^{-/-} 3^{-/-}$TKO mice were generated by systematically breeding the $\mathrm{Pim}^{-/-} 2^{-/-}$and $\mathrm{Pim} 2^{-/-} 3^{-/-}$double KO mice and were on FVB/J background. Pim TKO mice and WT controls were maintained in our specific pathogen-free animal facility. The genotype of each mouse used in the study was confirmed by PCR genotyping of tail DNA.

\section{FVB/J mice}

WT FVB/J transplant recipient mice were purchased from the Jackson Laboratory. All our studies were 
performed in accordance with Medical University of South Carolina Institutional Animal Care and Use Committee approved- procedures.

\section{Peripheral blood cell subset analysis}

Whole blood hematological parameters including white blood cell count, hemoglobin concentration, hematocrit, platelet count, mean corpuscular volume (MCV), and mean corpuscular hemoglobin $(\mathrm{MCH})$ were measured using Scil ABC plus hematology analyzer (scil animal care company Ltd.) as per the manufacturer's instructions. Peripheral blood cell subsets were quantified using flow cytometry as described previously [25]. Briefly, $50 \mu \mathrm{l}$ blood was stained with monoclonal antibodies against various cell subsets [APC- CD3 (145-2C11), APC-Cy7- CD4 (GK1.5), PE-Cy7-CD8 (53-6.7), FITCB220 (RA3-6B2), PE- Gr-1 (RB6-8C5)]. Equal volume $(50 \mu \mathrm{l})$ of Flow-Count fluorospheres (Beckman-Coulter) was added before flow cytometric analysis. The absolute cell counts were calculated using the following formula: Absolute count $($ cells $/ \mu \mathrm{L}$ blood $)=($ Total number of cells counted/Total number of fluorospheres counted) $\times$ Flow-Count fluorosphere concentration.

\section{Colony forming unit (CFU) assay}

CFU assays were performed in complete M3434 methylcellulose medium (Stem Cell Technologies) following the manufacturer's instructions. Briefly, BM cells from Pim TKO mice or WT controls were resuspended in complete M3434 medium and plated in 6-well plates at $1 \times 10^{5}$ cells/well. The assays were done in triplicate and the number of CFUs-GM, BFUs-E and CFUs-GEMM was counted at day 7 , day 9 and day 12 , respectively.

\section{In vivo BrdU incorporation assay}

In vivo BrdU incorporation was performed as described previously with minor modifications [32,33]. Briefly, mice were intraperitoneally injected with 2 doses (at 8 and 2 hours before sacrifice) of bromodeoxyuridine (5-bromo-2deoxyuridine [BrdU]; BD Biosciences) at $50 \mu \mathrm{g} / \mathrm{gram}$ of body weight. BM cells were then isolated and enriched for Lin $^{-}$cell population using lineage cell depletion kit (Miltenyi Biotec). At least $1.5 \times 10^{6} \mathrm{Lin}^{-} \mathrm{BM}$ cells were labeled with PE -conjugated anti- mouse Sca-1, APC- conjugated anti mouse c-Kit, PerCP-eFluor- conjugated anti mouse CD135, and eFluor 450- conjugated anti mouse CD34 antibodies, followed by fixation and staining with FITC-conjugated BrdU antibody (BrdU Flow Kit, BD Pharmingen), according to the manufacturer's protocol.

\section{Hematopoietic stem cell transplantation (HCT)}

For primary $\mathrm{HCT}, \mathrm{BM}$ cells were isolated from male TKO mice or age matched WT controls. The red blood cell (RBC)- depleted BM cells were injected (cell doses were indicated in the text) via tail-vein to lethally irradiated (11Gy) female FVB/J recipient mice. Animal survival was monitored daily. To determine hematological recovery, peripheral blood was collected from transplant recipient mice by retro-orbital sampling under anesthesia condition. Whole blood cell counts were measured using a Scil ABC plus hematology analyzer as per the manufacturer's instructions.

For secondary HCT, BM cells were obtained from primary transplanted recipient mice at 4 months post transplantation, and $1 \times 10^{7} \mathrm{BM}$ cells/recipient were injected into lethally irradiated female FVB/J mice. Male donor cell engraftment was measured.

For competitive repopulation assay, $5 \times 10^{5}$ male $\mathrm{BM}$ donor cells from Pim TKO mice or WT controls were mixed with $2 \times 10^{5}$ female competitive BM cells from FVB/J mice, and transplanted into lethally irradiated female FVB/J mice.

\section{Analysis of donor cell engraftment}

Male donor cell engraftment in female transplant recipients was determined as described $[13,16]$. Briefly, genomic DNA was extracted from $\mathrm{RBC}$ - lysed peripheral blood cells or BM cells using the DNeasy Kit (QIAGEN), and further purified using Ethanol precipitation method. Twenty ng of genomic DNA were mixed with SYBR Green PCR master mix reagents (Bio-Rad) and real time PCR was performed. Donor cell engraftment was estimated by percentage of male DNA calculated from the standard curve by PCR for sex-determining region Y (Zfy1) [16]. Bcl-2: 5'-AAGCTG TCACAGAGGGGCTA and 5'-CAGGCTGGAAGGAGA AGATG or Actin: 5'-TGTTACCAACTGGGACGACA and 5'- ACCTGGGTCATCTTTTCACG were used as reference genes.

\section{Cell sorting for $\mathrm{CD} 3 \mathrm{e}^{+} \mathrm{T}$ cells, $\mathrm{Gr}-1^{+}$granulocytes, and B220 ${ }^{+}$B cells}

Peripheral blood samples $(150 \mu \mathrm{l} /$ mouse $)$ were collected from each group (5 mice/group) at 4 months post competitive BM transplant and pooled. After RBC depletion, the leukocytes were stained with APC- conjugated anti mouse CD3e, PE conjugated anti mouse Gr-1 and FITC -conjugated anti mouse B220 antibodies and subjected to cell sorting on Mo-Flo sorter (DakoCytomation). Sorted CD3e ${ }^{+} \mathrm{T}$ cells, $\mathrm{Gr}-1^{+}$granulocytes and $\mathrm{B} 220^{+} \mathrm{B}$ cells were processed for genomic DNA isolation. Male donor cell engraftment in each cell subset was determined by real time PCR analysis as described above.

\section{In vitro culture of LSK cells and cell proliferation and apoptosis assay}

RBC-depleted total BM cells obtained from Pim TKO mice or WT control mice were first enriched for Lin $^{-}$cells by Lineage selection kit. Lin' cells were then 
stained with Scal-1 and c-Kit antibodies and sorted on flow cytometry for LSK cells. LSK cells $(6,000 /$ well) were cultured in StemSpan SFEM medium (StemCell Technologies) supplemented with $100 \mathrm{ng} / \mathrm{mL}$ of murine stem cell factor (SCF), murine Thrombopoietin (TPO), and murine Flt3 (all from Invitrogen) for 2 days. Cells were then stained with Aqua Live/dead fixable dye (Invitrogen) followed by fixation and permeabilization (BD Cytofix/Cytoperm kit) according to the manufacturer's instructions. The fixed cells were then stained with PE-Cy7-conjugated Ki-67 antibody and FITC-labeled caspase 3 antibody.

\section{Statistical analysis}

The values were reported as Mean \pm SEM of multiple experiments or Mean \pm SD from a representative experiment. Differences were analyzed by Student's $t$ test. $\mathrm{p}<0.05$ was regarded as significant.

\section{Competing interests}

The authors declare no competing financial interests.

\section{Authors' contribution}

NA performed research and analyzed data. ASK designed research. NA and YK designed research and wrote the paper. All authors reviewed and approved the final manuscript.

\section{Authors' information}

Ningfei An, PhD, is a postdoctoral research associate at the Medical University of South Carolina. Andrew Kraft, MD, is a professor of medicine and the director of the Hollings Cancer Center, Medical University of South Carolina. Yubin Kang, MD, is an assistant professor and a hematologist/ oncologist at the Hollings Cancer Center, Medical University of South Carolina.

\section{Acknowledgements}

We thank Richard Peppler at the HCC Flow Cytometry Core for performing flow cytometry analysis. We thank Dr. Woodrow J. Coker III for critical reading of the manuscript. This work is supported by MUSC Hollings Cancer Center Startup Fund, Hollings Cancer Center ACS IRG (YK), ASCO Conquer Cancer Foundation Career Development Award (YK), NIH 1 K08HL 103780$01 \mathrm{~A} 1$ (YK), and NIH 3P30CA138313-01S3. The content is solely the responsibility of the authors and does not necessarily represent the official views of the National Institutes of Health or other funding agents.

Received: 19 September 2012 Accepted: 25 January 2013

Published: 29 January 2013

\section{References}

1. Cuypers HT, Selten G, Quint W, Zijlstra M, Maandag ER, Boelens W, van Wezenbeek P, Melief C, Berns A: Murine leukemia virus-induced T-cell lymphomagenesis: integration of proviruses in a distinct chromosomal region. Cell 1984, 37(1):141-150.

2. Breuer ML, Cuypers HT, Berns A: Evidence for the involvement of pim-2, a new common proviral insertion site, in progression of lymphomas. EMBO J 1989, 8(3):743-748.

3. Mikkers H, Allen J, Knipscheer P, Romeijn L, Hart A, Vink E, Berns A: Highthroughput retroviral tagging to identify components of specific signaling pathways in cancer. Nat Genet 2002, 32(1):153-159.

4. Mikkers H, Nawijn M, Allen J, Brouwers C, Verhoeven E, Jonkers J, Berns A: Mice deficient for all PIM kinases display reduced body size and impaired responses to hematopoietic growth factors. Mol Cell Biol 2004, 24(13):6104-6115.

5. Brault L, Gasser C, Bracher F, Huber K, Knapp S, Schwaller J: PIM serine/ threonine kinases in the pathogenesis and therapy of hematologic malignancies and solid cancers. Haematologica 2010, 95(6):1004-1015.
6. Eichmann A, Yuan L, Breant C, Alitalo K, Koskinen PJ: Developmental expression of pim kinases suggests functions also outside of the hematopoietic system. Oncogene 2000, 19(9):1215-1224.

7. Macdonald A, Campbell DG, Toth R, McLauchlan H, Hastie CJ, Arthur JS: Pim kinases phosphorylate multiple sites on Bad and promote 14-3-3 binding and dissociation from Bcl-XL. BMC Cell Biol 2006, 7:1.

8. van Lohuizen M, Verbeek S, Krimpenfort P, Domen J, Saris C, Radaszkiewicz $T$, Berns A: Predisposition to lymphomagenesis in pim-1 transgenic mice: cooperation with c-myc and $\mathrm{N}$-myc in murine leukemia virus-induced tumors. Cell 1989, 56(4):673-682.

9. Allen JD, Verhoeven E, Domen J, van der Valk M, Berns A: Pim-2 transgene induces lymphoid tumors, exhibiting potent synergy with c-myc. Oncogene 1997, 15(10):1133-1141.

10. Laird PW, van der Lugt NM, Clarke A, Domen J, Linders K, McWhir J, Berns A Hooper M: In vivo analysis of Pim-1 deficiency. Nucleic Acids Res 1993, 21(20):4750-4755.

11. Hammerman PS, Fox CJ, Birnbaum MJ, Thompson CB: Pim and Akt oncogenes are independent regulators of hematopoietic cell growth and survival. Blood 2005, 105(11):4477-4483.

12. Grundler R, Brault L, Gasser C, Bullock AN, Dechow T, Woetzel S, Pogacic V, Villa A, Ehret $\mathrm{S}$, Berridge $\mathrm{G}$, et al: Dissection of PIM serine/threonine kinases in FLT3ITD-induced leukemogenesis reveals PIM1 as regulator of CXCL12-CXCR4mediated homing and migration. J Exp Med 2009, 206(9):1957-1970.

13. An N, Kang Y: Using quantitative real-time PCR to determine donor cell engraftment in a competitive murine bone marrow transplantation model. J Vis Exp 2013. In press.

14. Kang Y, Chen BJ, Deoliveira D, Mito J, Chao NJ: Selective enhancement of donor hematopoietic cell engraftment by the CXCR4 antagonist AMD3100 in a mouse transplantation model. PLoS One 2010, 5(6):e11316.

15. Adolfsson J, Borge OJ, Bryder D, Theilgaard-Monch K, Astrand-Grundstrom I, Sitnicka E, Sasaki Y, Jacobsen SE: Upregulation of Flt3 expression within the bone marrow Lin(-)Sca1(+)c-kit(+) stem cell compartment is accompanied by loss of self-renewal capacity. Immunity 2001, 15(4):659-669.

16. Byrne P, Huang W, Wallace VM, Shean MK, Zhang Z, Zhong Q, Theodossiou C, Blakesley H, Kolls JK, Schwarzenberger P: Chimerism analysis in sexmismatched murine transplantation using quantitative real-time PCR. Biotechniques 2002, 32(2):279-280-282-274, 286

17. Schuster JA, Stupnikov MR, Ma G, Liao W, Lai R, Ma Y, Aguila JR: Expansion of hematopoietic stem cells for transplantation: current perspectives. Exp Hematol Oncol 2012, 1(1):12.

18. Amson R, Sigaux F, Przedborski S, Flandrin G, Givol D, Telerman A: The human protooncogene product p33pim is expressed during fetal hematopoiesis and in diverse leukemias. Proc Natl Acad Sci U S A 1989, 86(22):8857-8861.

19. Hu YL, Passegue E, Fong S, Largman C, Lawrence HJ: Evidence that the Pim1 kinase gene is a direct target of HOXA9. Blood 2007, 109(11):4732-4738.

20. Aksoy I, Sakabedoyan C, Bourillot PY, Malashicheva AB, Mancip J, Knoblauch K, Afanassieff M, Savatier P: Self-renewal of murine embryonic stem cells is supported by the serine/threonine kinases Pim-1 and Pim-3. Stem Cells 2007, 25(12):2996-3004.

21. Pircher TJ, Zhao S, Geiger JN, Joneja B, Wojchowski DM: Pim-1 kinase protects hematopoietic FDC cells from genotoxin-induced death. Oncogene 2000, 19(32):3684-3692.

22. Nosaka T, Kitamura T: Pim-1 expression is sufficient to induce cytokine independence in murine hematopoietic cells, but is dispensable for BCRABL-mediated transformation. Exp Hematol 2002, 30(7):697-702.

23. Lilly M, Kraft A: Enforced expression of the Mr 33,000 Pim-1 kinase enhances factor-independent survival and inhibits apoptosis in murine myeloid cells. Cancer Res 1997, 57(23):5348-5355.

24. Mochizuki T, Kitanaka C, Noguchi K, Muramatsu T, Asai A, Kuchino Y: Physical and functional interactions between Pim-1 kinase and Cdc25A phosphatase. Implications for the Pim-1-mediated activation of the c-Myc signaling pathway. J Biol Chem 1999, 274(26):18659-18666.

25. Bachmann M, Kosan C, Xing PX, Montenarh M, Hoffmann I, Moroy T: The oncogenic serine/threonine kinase Pim-1 directly phosphorylates and activates the G2/M specific phosphatase Cdc25C. Int J Biochem Cell Biol 2006, 38(3):430-443.

26. Morishita D, Katayama R, Sekimizu K, Tsuruo T, Fujita N: Pim kinases promote cell cycle progression by phosphorylating and down-regulating p27Kip1 at the transcriptional and posttranscriptional levels. Cancer Res 2008, 68(13):5076-5085. 
27. Aho TL, Sandholm J, Peltola KJ, Mankonen HP, Lilly M, Koskinen PJ: Pim-1 kinase promotes inactivation of the pro-apoptotic Bad protein by phosphorylating it on the Ser112 gatekeeper site. FEBS Lett 2004, 571(1-3):43-49.

28. Gu JJ, Wang Z, Reeves R, Magnuson NS: PIM1 phosphorylates and negatively regulates ASK1-mediated apoptosis. Oncogene 2009, 28(48):4261-4271.

29. Zhang F, Beharry ZM, Harris TE, Lilly MB, Smith CD, Mahajan S, Kraft AS: PIM1 protein kinase regulates PRAS40 phosphorylation and mTOR activity in FDCP1 cells. Cancer Biol Ther 2009, 8(9):846-853.

30. Beharry Z, Mahajan S, Zemskova M, Lin YW, Tholanikunnel BG, Xia Z, Smith $C D$, Kraft AS: The Pim protein kinases regulate energy metabolism and cell growth. Proc Natl Acad Sci U S A 2011, 108(2):528-533.

31. Alvarado Y, Giles FJ, Swords RT: The PIM kinases in hematological cancers. Expert Rev Hematol 2012, 5(1):81-96.

32. Merchant A, Joseph G, Wang Q, Brennan S, Matsui W: Gli1 regulates the proliferation and differentiation of HSCs and myeloid progenitors. Blood 2010, 115(12):2391-2396.

33. Johnson SM, Torrice CD, Bell JF, Monahan KB, Jiang Q, Wang Y, Ramsey MR, Jin J, Wong KK, Su L, et al: Mitigation of hematologic radiation toxicity in mice through pharmacological quiescence induced by $C D K 4 / 6$ inhibition. J Clin Invest 2010, 120(7):2528-2536.

doi:10.1186/1756-8722-6-12

Cite this article as: An et al: Abnormal hematopoietic phenotypes in

Pim kinase triple knockout mice. Journal of Hematology \& Oncology 2013 6:12.

\section{Submit your next manuscript to BioMed Central and take full advantage of:}

- Convenient online submission

- Thorough peer review

- No space constraints or color figure charges

- Immediate publication on acceptance

- Inclusion in PubMed, CAS, Scopus and Google Scholar

- Research which is freely available for redistribution 